\title{
Beyond-mean-field crossover from one dimension to three dimensions in quantum droplets of binary mixtures
}

\author{
L. Lavoine and T. Bourdel * \\ Laboratoire Charles Fabry, UMR 8501, Institut d'Optique, CNRS, \\ Universit Paris-Saclay, Avenue Augustin Fresnel, 91127 Palaiseau CEDEX, France
}

(Dated: March 18, 2021)

\begin{abstract}
The existence of quantum droplets in binary Bose-Einstein condensate mixtures rely on beyondmean field effects, competing with mean-field-effects. Interestingly, the beyond-mean field effects change from repulsive in three dimension (3D) to attractive in 1D leading to drastically different behaviors. We quantitatively model quantum droplets in the beyond-mean-field crossover from 1D to $3 \mathrm{D}$ in the relevant case of an elongated harmonic trap and give realistic numbers for experimental realizations. We identify and quantify two main limiting factors: three-body losses and tiny energy scales. The crossover region is appealing as it offers a trade-off between these two main limitations opening the possibility of observing stable flat-top density profiles, a yet unobserved, characteristic feature of quantum droplets. It would permit testing beyond-mean-field theories to an unprecedented precision.
\end{abstract}

PACS numbers:

\section{INTRODUCTION}

Ultracold quantum gases are uniquely well controlled many-body systems [1]. Their diluteness permits accurate $a b$ initio theoretical treatment using zero range interaction. They are thus good candidates for tests of many-body theories. As an example, the energy of a zero temperature Bose gas can be calculated within the Bogoliubov theory beyond the usual mean-field approximation [2, 3]. Experimentally, there has been a quest for the measurement of these beyond mean-field corrections mostly by increasing $n a^{3}$, where $n$ is the density and $a$ is the scattering length [4-7].

Recently, it was discovered that quantum mixtures of two Bose-Einstein condensates with repulsive intraspecies interaction and attractive interspecies interactions permit a cancellation of the global mean-field interaction without a reduction of the magnitude of the beyond mean-field effects [8]. They can then play a dominant role in the dynamics of the system and compete with the reduced mean-field energy. In this context, quantum droplets, i.e. self-bound Bose-Einstein condensates due to beyond-mean field effects have been predicted [8] and experimentally observed [9-12]. The name droplet is given in analogy to liquid droplets which have similar properties such as a constant density profile although their stabilization mechanism is different. Quantum droplets were also observed in dipolar condensates where the magnetic interaction competes with the usual contact interaction [13] (see Refs [14, 15] and references therein for recent reviews on quantum droplets). Experimentally, the droplets are observed at high densities (typically $\sim 10^{21} \mathrm{~m}^{-3}$ ). Three-body losses thus play an important role in the droplet dynamics [16] and have

\footnotetext{
*Email: thomas.bourdel@institutoptique.fr
}

hindered the observation of the predicted characteristic flat-top density profile of a stable droplet.

Interestingly, the beyond-mean-field energy, which originates from the summation of the zero point energies of the Bogoliubov modes in the Lee-Huang-Yang description [17], strongly depends on the dimension of the systems with important consequences [18]. For example, the three-dimensional (3D) beyond-mean-field energy density is positive and scales as $n^{5 / 2}$, whereas the $1 \mathrm{D}$ beyondmean-field energy density is negative and scales as $n^{3 / 2}$. In the $1 \mathrm{D}$ case, a dominant beyond-mean-field energy is obtained at low density in contrast to the 3D case. Quantum droplets thus exist in both cases in however quite different conditions, requiring in particular an opposite sign of the mean-field interaction [19]. The beyond-mean-field energy density in the 1D-3D crossover was calculated predicting the existence of quantum droplets for any value and sign of the mean-field interaction term in elongated geometries [20, 21].

Experimentally, droplets in Bose-Bose mixtures have been observed not only in free-space [10] but also in cigar and pancake traps corresponding to quasi-1D [11] and quasi-2D situations [9], where the motion of particles is frozen in one or two directions. Nevertheless, the beyondmean-field effects were still in a $3 \mathrm{D}$ regime. This possibility of hybrid dimension comes from the different energy scales associated with the two excitation branches, which are relevant in droplets of binary mixtures, i.e. the low energy density branch (the two condensates oscillate in phase) and the high energy spin branch (the two condensates oscillate out of phase). The latter is responsible for the main beyond-mean field effects [21].

In this paper, we study the $1 \mathrm{D}-3 \mathrm{D}$ crossover for quasi$1 \mathrm{D}$ quantum droplets in the experimentally relevant case of a cigar-shaped harmonic trap. Our work crucially uses a calculation of the beyond-mean-field energy density, in which the radial wave-function is fixed to the radial harmonic oscillator one [20]. There is a smooth 
1D-3D crossover where the spin mode excitations and thus also the Lee-Huang-Yang energy density are progressively modified by the radial trapping. In the whole crossover, we study important experimental parameters such as the droplet density, size, binding energy or threebody loss rate as a function of the relevant dimensionless parameters. The paper first describes large bulk droplet properties and second introduces finite size effects within an extended Gross-Pitaevskii equation formalism. We study the ground state droplets with and without longitudinal trapping and find the conditions in which a flat-top density profile is expected. We also study the breathing mode frequency which gives the relevant time scale for an adiabatic preparation of quantum droplets. We find that the crossover regime offers an experimentally favorable trade-off between high three-body losses close to the $3 \mathrm{D}$ regime and tiny energy scales in the $1 \mathrm{D}$ regime.

\section{QUANTUM DROPLETS IN THE BULK}

We consider a mixture of two atomic Bose-Einstein condensates in states $|1\rangle$ and $|2\rangle$ (of equal mass $m$ for simplicity) and radially harmonically trapped with a frequency $\omega_{\perp} / 2 \pi$. We assume that we are in the quasi-1D regime such that the radial condensate wave-functions are Gaussian characterized by the harmonic oscillator size $a_{o h}=\sqrt{\hbar / m \omega_{\perp}}$, where $\hbar$ is the reduced Planck constant. The three relevant scattering lengths are $a_{11}>$ $0, a_{22}>0, a_{12}<0$ associated with the $1 \mathrm{D}$ coupling constants $g_{i j}=2 \hbar \omega_{\perp} a_{i j}$. We first consider the homogenous case with the two $1 \mathrm{D}$ densities $n_{1}, n_{2}$ and the total density $n=n_{1}+n_{2}$.

The mean field energy density can be written as:

$$
\begin{gathered}
E_{M F}=g_{11} n_{1}^{2} / 2+g_{22} n_{2}^{2} / 2+g_{12} n_{1} n_{2} \\
E_{M F}=\frac{\left(\sqrt{g_{11}} n_{1}-\sqrt{g_{22}} n_{2}\right)^{2}}{2}+\frac{g \delta g\left(\sqrt{g_{11}} n_{2}+\sqrt{g_{22}} n_{1}\right)^{2}}{\left(g_{11}+g_{22}\right)^{2}} \\
\quad \text { with } \delta g=g_{12}+\sqrt{g_{11} g_{22}} \text { and } g=\sqrt{g_{11} g_{22}}
\end{gathered}
$$

In the vicinity of the mean-field collapse $\delta g / g \ll 1$, the first term is much larger than the second one. The system thus minimizes its mean-field energy by locking the two densities such that $\sqrt{g_{11}} n_{1}=\sqrt{g_{22}} n_{2}$. In this situation, the mean-field energy density reduces to

$$
\begin{gathered}
\frac{E_{M F}}{\hbar \omega_{\perp}}=\frac{2 a \delta a}{\left(\sqrt{a_{11}}+\sqrt{a_{22}}\right)^{2}} n^{2} \\
\text { with } \delta a=a_{12}+\sqrt{a_{11} a_{22}} \text { and } a=\sqrt{a_{11} a_{22}}
\end{gathered}
$$

This equation can be written in a more convenient form

$$
\frac{E_{M F}}{\hbar \omega_{\perp}}=\delta a^{\prime} \lambda \kappa^{2} / 2 a
$$

with $\kappa=n a, \lambda=a / a_{o h}$, and $\delta a^{\prime}=\frac{4 \delta a}{\lambda\left(a_{11}^{1 / 2}+a_{22}^{1 / 2}\right)^{2}}$ being a dimensionless parameter characterizing the mean-field interaction.
The beyond-mean-field energy density has been calculated in two limits depending on the value of $\kappa$. For $\kappa \gg 1$ corresponding to large densities such that the spin healing length is smaller than $a_{o h}$, one can make a local density approximation along the radial Gaussian density profile and integrate the usual 3D beyond-mean-field expression [20]:

$$
\frac{E_{B M F}^{3 D}}{\hbar \omega_{\perp}}=\frac{\lambda}{a} \frac{512}{75 \pi} \kappa^{5 / 2} .
$$

In the opposite limit $\kappa \lesssim 1$, the summation over the radial oscillation modes has been performed in order to calculate the beyond-mean field energy density of a quasi-1D Bose-Einstein condensate in the 1D-3D crossover [20]. Interestingly, the result can also be used for the spin modes in a Bose-Bose mixture, giving the following beyondmean field contribution.

$$
\begin{gathered}
\frac{E_{B M F}^{c}}{\hbar \omega_{\perp}}=\frac{\lambda}{a} f(\kappa), \text { with } \\
f(\kappa)=\frac{C_{1 D}^{h}}{\sqrt{2}} \kappa^{2}-\frac{4 \sqrt{2}}{3 \pi} \kappa^{3 / 2}+\frac{4 \sqrt{2} \ln \left(\frac{4}{3}\right)}{\pi} \kappa^{5 / 2}+B_{1 D}^{h} \kappa^{3} .
\end{gathered}
$$

The first term in $f(\kappa)$ is a mean-field correction due to the confinement-induced resonance $\left(C_{1 D}^{h} \approx 1.4603\right)$. The second term is the beyond mean-field contribution of a purely one-dimensional system [18], which dominates at small $\kappa$. The third and fourth terms are corrections for higher values of $\kappa\left(B_{1 D}^{h} \approx 1.13\right.$ [22]). For $\kappa \sim 1$, the beyond-mean-field energy density cannot be written in a simple form but one can interpolate between the two previous expressions with a relatively good accuracy.

In the two cases, one can simply minimize the energy per particles $E=\left(E_{M F}+E_{B M F}\right) / n$ as a function of $\kappa$ in order to find the equilibrium density in the bulk, i.e. neglecting the kinetic energy. We plot the resulting value of $\kappa$ as a function of the mean-field parameter $\delta a^{\prime}$ (see Fig. 1). Note that in the 3D beyond-mean field case, the minimization leads to a non-zero density only for attractive mean-field interaction which compensates a repulsive beyond-mean-field term (red curve). In contrast, the crossover expression leads to the existence of a finite density droplet for any value of the mean-field parameter (black curve). For large and negative meanfield parameter $\delta a^{\prime}$, we find $\kappa \gg 1$ and the 3D expression is the valid one. For $\delta a^{\prime} \gtrsim-3$, the crossover expression finds $\kappa \lesssim 1$ and it is thus valid. By interpolating the two results from their validity region, we find droplets for any value of the mean-field interaction and an approximated value of $\kappa$ in the whole crossover. The equilibrium density is drastically reduced when increasing the mean-field interaction parameter.

We also plot the energy per particle in Fig. 2. It is negative as a droplet is a self bound object. One can interpolate between the two solid curves in order to find its behavior for any parameter $\delta a^{\prime}$. The binding energy of the droplet significantly decreases as one goes toward the 


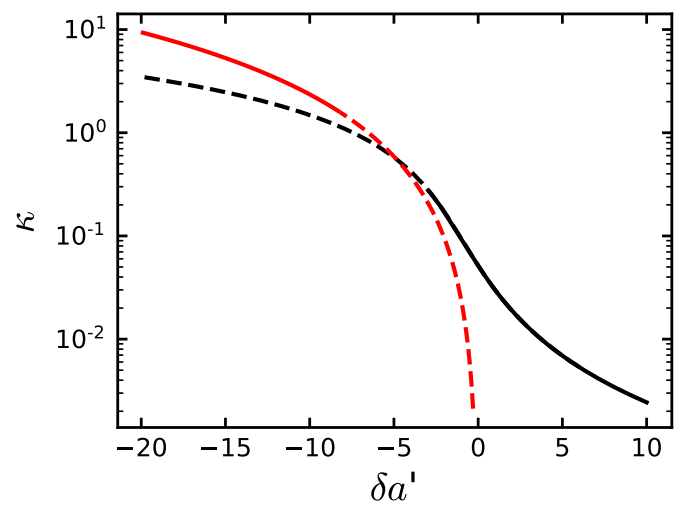

FIG. 1: (Color online) Dimensionless density $\kappa$ in a bulk quantum droplet as a function of the mean-field parameter $\delta a^{\prime}$. The black (red) curve corresponds to the 1D-3D crossover (3D) beyond-mean-field model. The curves are dashed in the regions where the theory is expected not to be valid.

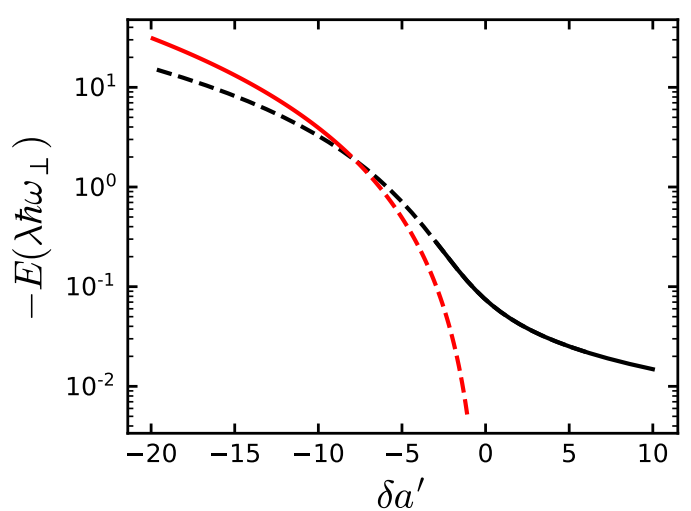

FIG. 2: (Color online) Energy per particle in the bulk. The black (red) curve corresponds to the energy minimization using the 1D-3D crossover (3D) formula. The curves are dashed in the regions where the corresponding theory is expected not to be valid.

1D regime. The relevant scaling for the binding energy $\lambda \hbar \omega_{\perp} \propto \omega_{\perp}^{3 / 2}$ shows the influence of the radial confinement.

In this context, we can thus wonder whether the increased lifetime of the droplet due to reduced densities and three-body loss rates is sufficient to counterbalance its reduced energy scale and thus increased time scale. The three-body loss rate is characterized by a threebody loss coefficient $K_{3}$. By integration over the Gaussian radial profile, we find the loss rate $\frac{1}{N} \frac{\partial N}{\partial t}=-\Gamma=$ $-K_{3} \frac{\kappa^{2}}{3 \pi^{2} a_{o h}^{4} a^{2}}$. We then plot the dimensionless ratio of the droplet binding energy to the three-body loss rate, which is a measure of the relative importance of losses as compared with the droplet intrinsic time scale (see Fig. 3). Small values imply that losses play an important role in the droplet dynamics [16]. We find that it is indeed fa- vorable to move toward $\delta a^{\prime}>0$ in order to minimize the relative effect of losses. Note that the ratio is plotted in units of the dimensionless quantity $\hbar a^{3} a_{o h} /\left(m K_{3}\right)$ which reveals the importance of the different parameters. In particular, we find that reducing the confinement $\omega_{\perp}$ is favorable to reduce the relative role of losses in the droplet dynamics despite the reduction in the droplet binding energy.

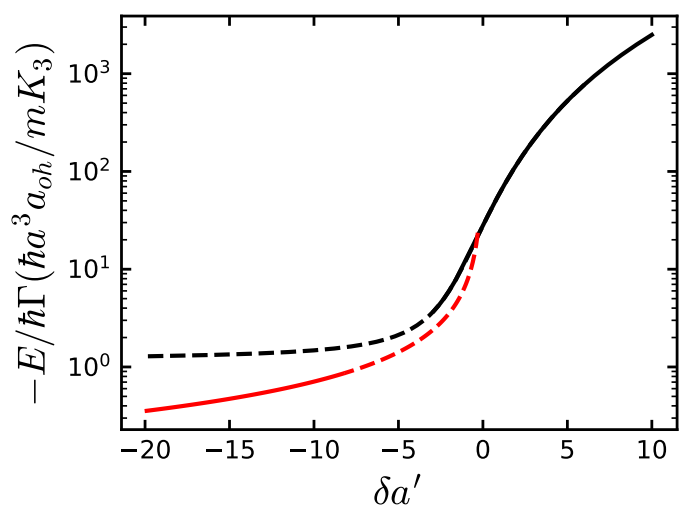

FIG. 3: (Color online) Ratio of the binding energy per particle to the three-body loss rate in the beyond-mean-field 1D-3D crossover. Larger ratios are favorable to minimize the relative effect of losses.

We now discuss experimentally realistic numbers. We consider droplets made of potassium-39 in the second and third spin states around $56.8 \mathrm{G}$ as used in previous experiments. In this case $a_{11} \approx 33 a_{0}, a_{22} \approx 84 a_{0}$, and $a_{12} \approx 53 a_{0}$, where $a_{0}$ is the Bohr radius. The meanfield parameters $\delta a$ and $\delta a^{\prime}$ can be varied around zero by slight adjustment of the magnetic field. As an example, we take $\omega_{\perp} / 2 \pi=500 \mathrm{~Hz}$ and the energy scale $\lambda \hbar \omega_{\perp} \approx(2 \pi \hbar) \times 2 \mathrm{~Hz}$ is relatively low. For $\delta a=-5 a_{0}$, a value close to the experimental ones [9-11], $\delta a^{\prime}=-20$ and the binding energy per particle $\sim(2 \pi \hbar) \times 60 \mathrm{~Hz}$ is such that droplet physics can be observed on a time scale of tens of milliseconds [11]. With an effective three-body loss rate coefficient $K_{3}=1.4 \times 10^{-40} \mathrm{~m}^{6} . \mathrm{s}^{-1}$ that can be assumed to be constant in the relevant region of the magnetic field $[11,23]$, the loss rate is $\sim 80 \mathrm{~s}^{-1}$ and losses play a significant role in the droplet dynamics.

When one goes toward the 1D regime, the energy scale decreases rapidly. Nevertheless, the loss rate decreases even more rapidly and the crossover regime is an adequate region to look for more stable droplets. For example at $\delta a^{\prime}=0$, where the droplet is solely stabilized by the peculiar density dependence of the beyond-mean field energy as a function of density [20], the droplet density is reduced by a factor $\sim 100$ and three-body losses are then negligible. The energy per particle is then of the order of $\sim(2 \pi \hbar) \times 0.2 \mathrm{~Hz}$. Note that such a low energy scale implies long experimental times as well as a control of the trap parameters such as its longitudinal flatness to an extreme precision. 


\section{QUANTUM DROPLETS IN A FINITE SYSTEM}

Whereas the above discussion focused on the properties of droplets in the bulk, we now turn to the question of finite atom numbers and finite sizes in quasi-1D droplets in the beyond-mean-field crossover from $1 \mathrm{D}$ to $3 \mathrm{D}$. One of our goals is to identify realistic parameters where a stable flat-top droplet density profile could be observed.

\section{A. Extended Gross-Pitaevskii equation}

In the same spirit as before, we suppose that the spin modes are unpopulated such that the ratio of densities between the two spin-states is fixed. Within this assumption the system can be described by a single wavefunction $\psi(x, t)$ solution of the following extended GrossPitaevskii equation:

$i \hbar \frac{\partial \psi}{\partial t}=-\frac{\hbar^{2}}{2 m} \frac{\partial^{2} \psi}{\partial x^{2}}+\hbar \omega_{\perp}\left(\delta a^{\prime} \lambda\left|\psi^{2}\right|+\lambda g(\kappa)\right) \psi+\frac{1}{2} m \omega_{\|}^{2} x^{2} \psi$

where $g(\kappa)=\frac{\partial f(\kappa)}{\partial \kappa}$ is the beyond-mean-field interaction potential.

This equation can be written in dimensionless units using the following scalings

$$
\begin{gathered}
t=t_{0} t^{\prime}=\frac{1}{\lambda \omega_{\perp}} t^{\prime} \\
x=x_{0} x^{\prime}=\frac{a_{\perp}}{\lambda^{1 / 2}} x^{\prime} \\
\psi=\psi_{0} \psi^{\prime}=\frac{1}{a^{1 / 2}} \psi^{\prime} \\
i \frac{\partial \psi^{\prime}}{\partial t^{\prime}}=-\frac{1}{2} \frac{\partial^{2} \psi^{\prime}}{\partial x^{\prime 2}}+\left(\delta a^{\prime}\left|\psi^{\prime 2}\right|+g\left(\left|\psi^{\prime 2}\right|\right)\right) \psi^{\prime}+\frac{1}{2} \frac{\omega_{\|}^{2}}{\omega_{\perp}^{2} \lambda^{2}} x^{\prime 2} \psi^{\prime}
\end{gathered}
$$

with the normalization condition $\int\left|\psi^{\prime 2}\right| \mathrm{d} x^{\prime}=N^{\prime}=$ $N \lambda^{3 / 2}$. In addition to this rescaled atom number, there are two dimensionless parameters: $\delta a^{\prime}$ for the mean-field interaction and $\frac{\omega_{\|}}{\omega_{\perp} \lambda}$ for the trapping potential.

\section{B. Ground state solutions}

We find the droplet ground state using the split step Fourier method and imaginary time evolution. We first study the solution with no longitudinal trapping $\left(\omega_{\|}=\right.$ $0)$. Remarkably we always find a self-bound solution for all parameters. This is in contrast to the $3 \mathrm{D}$ situation where a minimal atom number is needed to form a droplet. We now plot the ground state density profiles for two different atom numbers $N^{\prime}=0.3$ and $N^{\prime}=3$ and mean-field interaction parameters in the crossover $\delta a^{\prime}=-$ $6.7,0$, and 6.7 (corresponding to $\delta a / a_{0}=-1.6,0$, and 1.6 , for the previously given experimental parameters) in
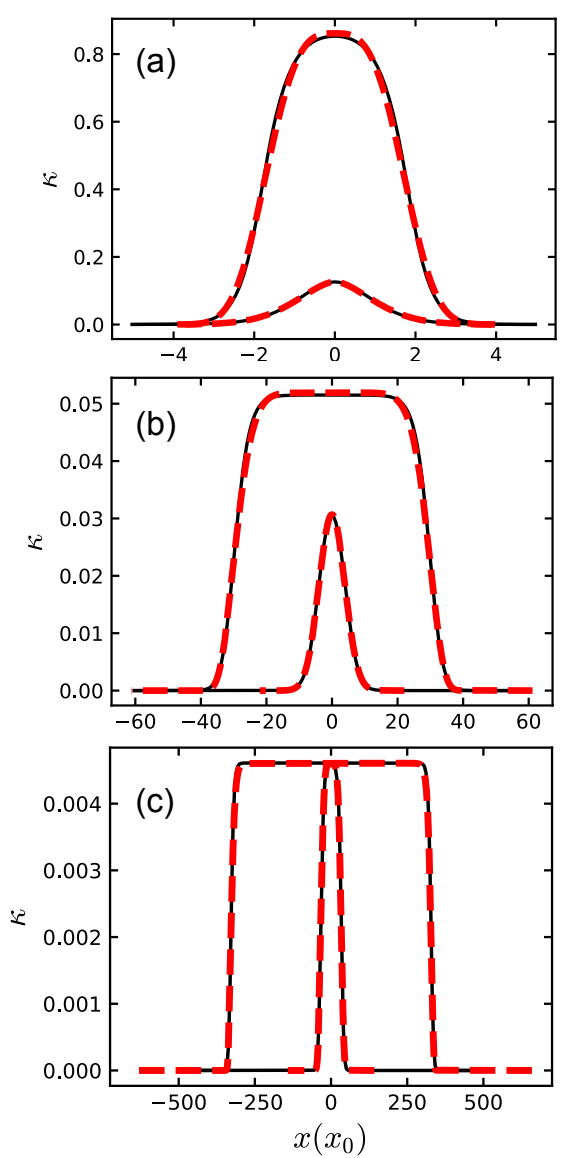

FIG. 4: (Color online) Quantum droplet density profiles in the absence of longitudinal trap $\left(\omega_{\|}=0\right)$ for $N^{\prime}=0.3$ and $N^{\prime}=3$ for three values of $\delta a^{\prime}$. (a): $\delta a^{\prime}=-6.7$. (b): $\delta a^{\prime}=0$. (c): $\delta a^{\prime}=6.7$. Please note, the different scales both in $x$ and in $\kappa$ in (a)-(c). The solid black curves correspond to the exact minimization of the extended Gross-Pitaevskii equation in the $1 \mathrm{D}-3 \mathrm{D}$ crossover using imaginary time evolution. The superimposed dashed red curves are the results using the two parameter ansatz presented in the text.

Fig. 4. For large atom numbers $N^{\prime} \geq 3$, the droplet profile exhibits a flat-top profile corresponding to the bulk solution whose edges are rounded because of the kineticenergy term. The droplet density gets smaller and its size larger as $\delta a^{\prime}$ goes from negative to positive values. For small atom numbers $N^{\prime} \leq 0.3$, the droplets do not show an extended flat region and the kinetic energy plays a dominant role.

For the realistic experimental parameters chosen previously, $N^{\prime}=1$ corresponds to 3800 atoms and the quasi$1 \mathrm{D}$ situation offers the possibility of saturating a droplet with realistic atom numbers. This is in contrast to the quasi-2D and even more the 3D droplets where the critical atom number to reach a flat top can be too high especially for low values of $|\delta a|$, which are favorable to reduce the role of three-body losses [8]. 
Another way to get an idea of the density profiles is to minimize the energy of an ansatz wavefunction. Here, an appropriate choice for the density profile is

$$
n(\sigma, r)=\frac{N}{2 \sigma \Gamma(1+1 / 2 r)} \exp \left(-\left(\frac{x^{2}}{\sigma^{2}}\right)^{r}\right) .
$$

This two-parameter function has the ability to interpolate between a peaked density profile for low values of $r$, a Gaussian density profile for $r=1$ and a flat-top density profile for $r \gg 1$. Its typical width is given by $\sigma$. It has the great advantage that the different energy terms are analytical and can be simply written in terms of the $\Gamma$ function. The energy minimization is then straightforward. In Fig. 4, it is obvious that the ansatz minimization method is able to approximate the exact ground state.

\section{Maximum density and RMS size: Scalings}
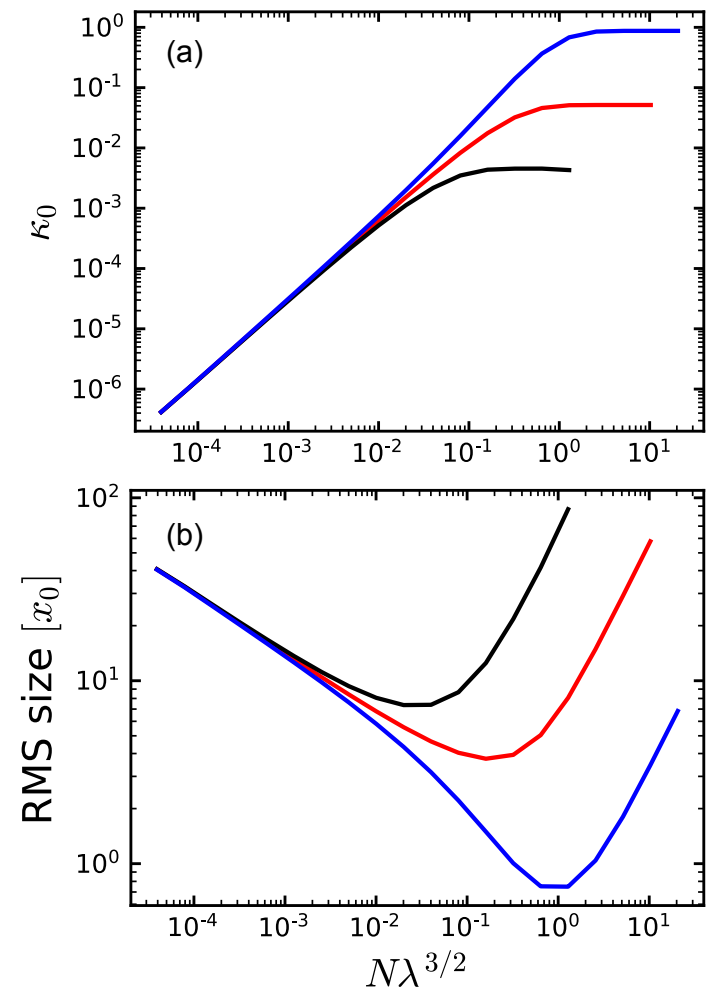

FIG. 5: (Color online) Maximum rescaled density $\kappa_{0}$ (a) and RMS size (b) of a droplet as a function of the atom number in the absence of a longitudinal trap $\left(\omega_{\|}=0\right)$. The three curves correspond to three values of the mean-field parameters $\delta a^{\prime}$ : black, $\delta a^{\prime}=6.7$; red (middle curve), $\delta a^{\prime}=0$; blue, $\delta a^{\prime}=-6.7$.

We now plot the maximum rescaled density $\kappa_{0}$ and the root-mean-square (RMS) size of the ground-state profile as a function of the atom number for $\delta a^{\prime}=-6.7,0$, and 6.7 (see Fig. 5). In all cases, the density increases with the atom number until it reaches a saturation value corresponding to the bulk density, when the droplet exhibits a flat-top profile. It also appears that a lower atom number is necessary to reach a flat-top droplet as one moves toward the 1D regime. The ground-state size first decreases as the atom number increases and then increases when a flat-top droplet is formed.

For the above figures, one can extract scalings in different regimes. For low atom numbers, all three curves are superimposed and $\kappa_{0} \ll 1$. The dominant energy terms are the $1 \mathrm{D}$ beyond-mean-field attractive terms and the kinetic energy. The mean-field term is negligible. In this case, the size scales as $N^{\prime-1 / 3}$ and the density scales as $N^{2 / 3}$. For a large atom number, the ground-state is a flat-top quantum droplet. The dominant energies are then the mean-field and beyond mean-field terms. The kinetic energy can be neglected in the analysis. In this case, the size simply scales as $N^{\prime}$ as the density is fixed. For attractive mean-field $\delta a^{\prime}<0$, there exists an intermediate atom number region in which the solution corresponds to a standard mean-field bright soliton [11, 24, 25] for which the size scales as $N^{\prime-1}$ and the density scales as $N^{\prime 2}$.

\section{Trapped case}

We now turn to the trapped case. As an example, we choose $\frac{\omega_{\|}}{\omega_{\perp} \lambda}=5 \times 10^{-3}$ (which corresponds to $w_{\|} / 2 \pi=0.01 \mathrm{~Hz}$, for the previously chosen parameters) and find the ground state by imaginary time evolution (see Fig. 6). For intermediate atom number, the trap has no effect. It corresponds to a regime where the trap potential energy is negligible as compared with the other energy scales. For low atom number, the size reaches a plateau in contrast to the untrapped case. This corresponds to a situation where the gas can be considered as non-interacting and the condensates occupy the ground state of the longitudinal harmonic trap.

At high atom numbers, the trap prevents the droplet from growing in size at a constant density as observed in the absence of a longitudinal trap. In this regime, the kinetic energy can be neglected and the density profile can be found in an approximation analogous to the Thomas-Fermi approximation. A local chemical potential is then directly linked to the density through the homogenous equation of state. In the $3 \mathrm{D}$ case, this was presented in [26]. The entrance in this last regime can be simply estimated by comparing the bulk energy per particle to the potential energy given by the RMS size of the droplet. The value of $N^{\prime}$ where this happens drastically depend on $\delta a^{\prime}$. As an example, density profiles for $N^{\prime}=10$ are plotted in figure 7 for different values of $\delta a^{\prime}$. The profile indeed resembles a Thomas-Fermi profile for $\delta a^{\prime}=6.7$ with dominant mean-field and potential energies. Oppositely, a flat-top droplet profile with negligible trap influence is found for $\delta a^{\prime}=-6.7$. For $\delta a^{\prime}=0$, the situation is intermediate. Our results show that even a 

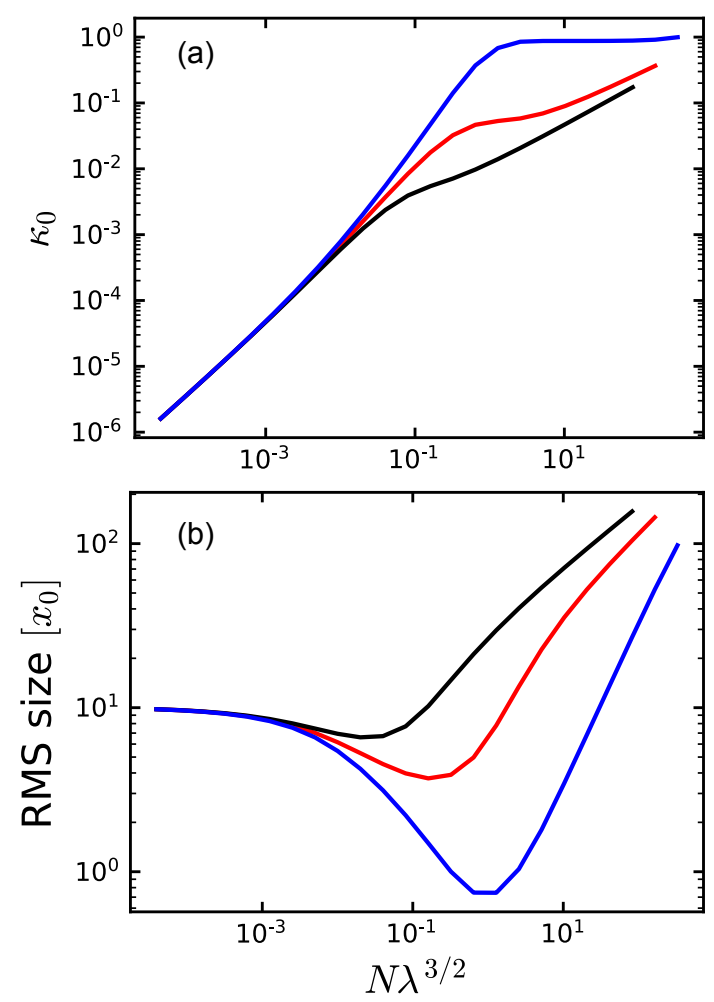

FIG. 6: (Color online) Maximum rescaled density $\kappa_{0}(\mathrm{a})$ and RMS size (b) as a function of the atom number in the presence of a longitudinal harmonic trap $\frac{\omega_{\|}}{\omega_{\perp} \lambda}=5 \times 10^{-3}$. The three curves follow the same coding as in Fig. 5 .

minute axial confinement can greatly affect the droplet shape in particular for $\delta a \gtrsim 0$ where the droplet energy scale is especially low.

\section{E. Breathing mode}

We now turn to the study of excitations of the quasi$1 \mathrm{D}$ droplets in the absence of a longitudinal trap. They can be studied by real time integration of the extended Gross-Pitaevskii equation. More specifically, we start from a situation close from equilibrium by rescaling the first computed ground state wave-function by a coefficient of 1.01 [19]. After this modification, we study in particular, the evolution of the RMS size of the droplet as a function of time and extract its main (lowest) oscillation frequency, which is plotted in Fig. 8. Interestingly, we find that these small breathing oscillations are essentially undamped. This can be expected as their frequency remains below the particle emission threshold for all parameters. High frequency modes can evaporate but there are only weakly excited in our excitation scheme [8]. This behavior is in contrast to the $3 \mathrm{D}$ situation, where there is a region just above the critical atom number where the droplets quickly evaporate to their ground state [8]. Importantly, the excitation mode period gives
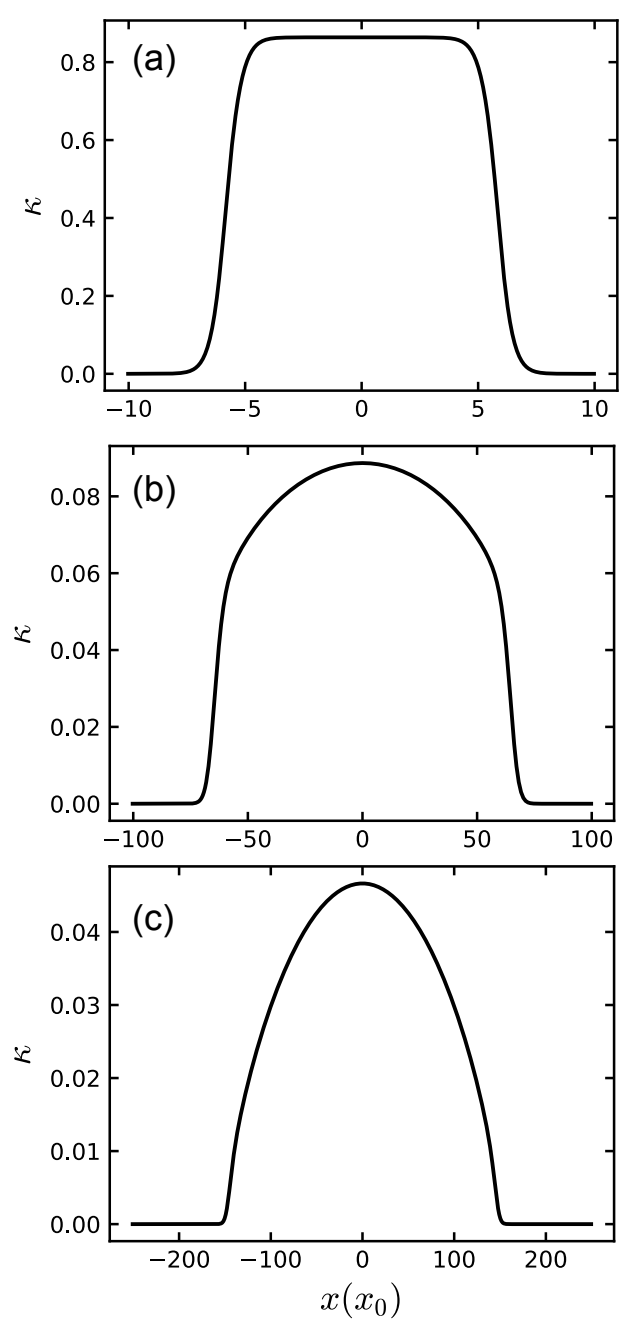

FIG. 7: (Color online) Droplet profiles in the presence of a longitudinal confinement $\frac{\omega_{\|}}{\omega_{\perp} \lambda}=5 \times 10^{-3}$ for $N^{\prime}=10$ and three different values of $\delta a^{\prime}$. (a): $\delta a^{\prime}=-6.7$. (b): $\delta a^{\prime}=0$. (c): $\delta a^{\prime}=6.7$.

a time scale in which a droplet can be prepared in an adiabatic (quasi-static) way by time variation of the experimental parameters.

We find that the breathing mode frequency first increases with the atom number as can be expected from an increasing binding energy. It then reaches a maximum close to the point where the droplet becomes flat topped. Then, it decreases when the droplet size increases. This observed behavior is qualitatively similar to the behavior previously predicted in the pure $1 \mathrm{D}$ regime $\left(\delta a^{\prime}>0\right)[19]$ but quantitatively differs. For an increasing value of $\delta a^{\prime}$, the maximum frequency takes significantly lower values. For the above mentioned parameters in potassium-39 mixtures, the frequency scale $\lambda \omega_{\perp} / 2 \pi=2 H z$ is already quite low and the droplet breathing mode frequency appears to be reasonably large only in the attractive mean field region. An adiabatic preparation of a ground state (unexcited) flat-top density droplet by a slow sweep of 


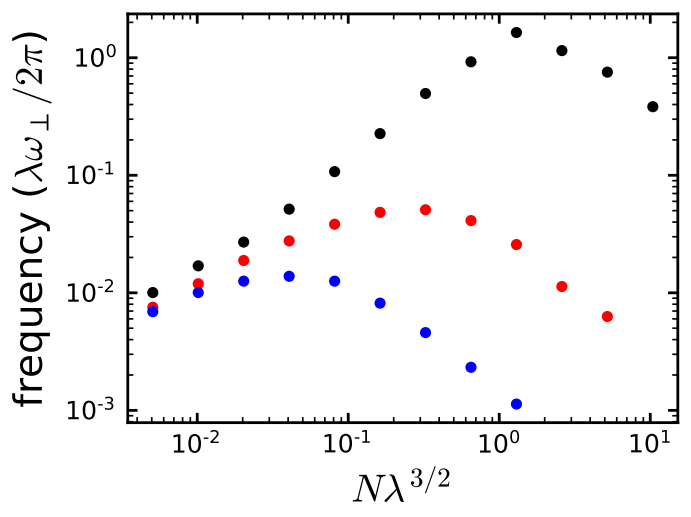

FIG. 8: (Color online) Frequency of the breathing mode as a function of the rescaled atom number for three values of the mean-field parameter $\delta a^{\prime}$ : black top points, $\delta a^{\prime}=-6.7$; red middle points, $\delta a^{\prime}=0$; blue lower points, $\delta a^{\prime}=6.7$.

the parameters seems challenging. Creating the droplet already close to its stable shape by releasing the gas from a longitudinal box potential appears to be a more realistic solution to avoid too strong excitations.

\section{CONCLUSIONS}

In a mixture of two Bose-Einstein condensates with repulsive intraspecies interaction and attractive interspecies interaction, we have studied quantum droplets in the 1D-3D crossover for the beyond-mean-field energy, which changes from positive in $3 \mathrm{D}$ to negative in $1 \mathrm{D}$. The equilibrium density is found to decrease rapidly when moving toward the $1 \mathrm{D}$ regime, which would be experimentally favorable in order to reduce the role of threebody losses. This reduction comes with a reduction in the typical energy scale of the droplets imposing severe experimental constrains on the control of the residual potential such as the longitudinal trapping or the preparation of the droplets close to their ground state. Nevertheless, stable quasi-1D quantum droplets with a characteristic flat-top profile should be realistically observable with a $\delta a^{\prime}$ that is negative and of the order of a few units in potassium 39 experiments [9-11]. The conditions would be even more favorable for low loss mixtures such as Rb-K [12].

The realization of quasi-1D droplets in the beyondmean-field crossover from $1 \mathrm{D}$ to $3 \mathrm{D}$ would allow precise studies of the beyond-mean-field effects in Bose gases. Effects beyond the standard Lee-Huang-Yang energy used in this paper will appear [27], either because of higherorder terms in the density expansion [28], because of finite-range interacting potentials $[29,30]$, or because of temperature effects [31].

\section{ACKNOWLEDGEMENTS}

We thank D. Petrov for inspiring discussions and A. Hammond for his careful rereading. This research has been supported by CNRS, Minstère de l'Enseignement Supérieur et de la Recherche, Labex PALM, Region Ilede-France in the framework of DIM Sirteq, Universit Paris-Saclay in the framework of IQUPS, ANR Droplets Project No. 19-CE30-0003-01, and the Simons Foundation (Award No. 563916: Localization of Waves).
[1] I. Bloch, J. Dalibard, and S. Nascimbène, Nature Physics 8, 267 (2012).

[2] T.D. Lee and C.N. Yang, Phys. Rev. 105, 1119 (1957).

[3] L. Pitaevskii and S. Stringari, Bose-Einstein Condensation and Superfluidity, Oxford University Press, Oxford, 2016.

[4] S. B. Papp, J. M. Pino, R. J. Wild, S. Ronen, C. E. Wieman, D. S. Jin, and E. A. Cornell, Phys. Rev. Lett. 101, 135301 (2008).

[5] Y. I. Shin, A. Schirotzek, C. H. Schunck, and W. Ketterle, Phys. Rev. Lett. 101, 070404 (2008).

[6] N. Navon, S. Piatecki, K. Günter, B. Rem, T. C. Nguyen, F. Chevy, W. Krauth, and C. Salomon, Phys. Rev. Lett. 107, 135301 (2011).

[7] R. Lopes, C. Eigen, N. Navon, D. Clément, R.P. Smith, and Z. Hadzibabic, Phys. Rev. Lett. 119, 190404 (2017).

[8] D.S. Petrov, Phys. Rev. Lett. 115, 155302 (2015).

[9] R. Cabrera, L. Tanzi, J. Sanz, B. Naylor, P. Thomas, P. Cheiney, and L. Tarruell, Science 359, 301 (2018).

[10] G. Semeghini, G. Ferioli, L. Masi, C. Mazzinghi, L. Wolswijk, F. Minardi, M. Modugno, G. Modugno, M. Inguscio, and M. Fattori, Phys. Rev. Lett. 120, 235301 (2018).

[11] P. Cheiney, C. R. Cabrera, J. Sanz, B. Naylor, L. Tanzi, and L. Tarruell, Phys. Rev. Lett. 120, 135301 (2018).

[12] C. D'Errico, A. Burchianti, M. Prevedelli, L. Salasnich, F. Ancilotto, M. Modugno, F. Minardi, and C. Fort, Phys. Rev. Res. 1, 033155 (2019).

[13] I. Ferrier-Barbut, H. Kadau, M. Schmitt, M. Wenzel, and T. Pfau, Phys. Rev. Lett. 116, 215301 (2016).

[14] Z.-H. Luo, W. Pang, B. Liu, Y.-Y. Li, B.A. Malomed, Front. Phys. 16, 32201 (2021).

[15] F. Böttcher, J.-N. Schmidt, J. Hertkorn, K.S.H. Ng, S.D. Graham, M. Guo, T. Langen, T. Pfau, Rep. Prog. Phys. 84, 012403 (2021).

[16] G. Ferioli, G. Semeghini, S. Terradas-Briansó, L. Masi, M. Fattori, and M. Modugno, Phys. Rev. Research 2, 013269 (2020).

[17] T.D. Lee, K.Huang and C.N. Yang, Phys. Rev. 106,1135 (1957).

[18] D. S. Petrov and G. E. Astrakharchik, Phys. Rev. Lett. 117, 100401 (2016).

[19] G. E. Astrakharchik and B. A. Malomed, Phys. Rev. A 98, 013631 (2018)

[20] T. Ilg, J. Kumlin, L. Santos, and D. S. Petrov, and, H. P. Büchler, Phys. Rev. A 98, 051604(R) (2018).

[21] P. Zin, M. Pylak, T.Wasak, M. Gajda, and Z. Idziaszek, 
Phys.Rev. A 98, 051603(R) (2018).

[22] There is a misprint in Ref. [20] for the value of $B_{1 D}^{h}$. L. Santos (Private communication).

[23] The given effective value of $K_{3}$ takes into account a $1 / 6$ factor due to the absence of Bose enhancement as compared with the thermal loss coefficient and also includes the fact that only a fraction of the atoms are in the lossy state $|2\rangle$.

[24] L. Khaykovich, F. Schreck, G. Ferrari, T. Bourdel, J. Cubizolles, L. D. Carr, Y.Castin, and C. Salomon, Science 296, 1290 (2002).

[25] K. E. Strecker, G. B. Patridge, A. G. Truscott, and R. G. Hulet, Nature (London) 417, 150 (2002).

[26] N.B. Jorgensen, G.M. Bruun, and J.J. Arlt Phys. Rev.
Lett. 121, 173403 (2018).

[27] F. Böttcher, M. Wenzel, J.-N. Schmidt, M. Guo, T. Langen, I. Ferrier-Barbut, T. Pfau, R. Bombin, J. SánchezBaena, J. Boronat, and F. Mazzanti, Phys. Rev. Research 1, 033088 (2019).

[28] M. Ota, G.E. Astrakharchik, SciPost Phys. 9, 020 (2020).

[29] V. Cikojević, L.V. Markić, G. E. Astrakharchik, and J. Boronat, Phys. Rev. A 99, 023618 (2019).

[30] V. Cikojević, L.V. Markić, M. Pi, M. Barranco, and J. Boronat, Phys. Rev. A 102, 033335 (2020).

[31] G. De Rosi, P. Massignan, M. Lewenstein, and G.E. Astrakharchik, Phys. Rev. Research 1, 033083 (2019). 